PROCEEDINGS OF THE

AMERICAN MATHEMATICAL SOCIETY

Volume 137, Number 8, August 2009, Pages 2685-2696

S 0002-9939(09)09812-8

Article electronically published on February 3, 2009

\title{
REALIZATION OF NONSTRICT MATRIX NEVANLINNA FUNCTIONS AS WEYL FUNCTIONS OF SYMMETRIC OPERATORS IN PONTRYAGIN SPACES
}

\author{
JUSSI BEHRNDT
}

(Communicated by Marius Junge)

\begin{abstract}
Matrix-valued Nevanlinna functions with possibly noninvertible imaginary part are realized as $Q$-functions or Weyl functions of symmetric operators in Pontryagin spaces. The functions are decomposed into a constant part, which gives rise to a realization in a finite dimensional Pontryagin space $\mathcal{K}$, and a strict or uniformly strict part, which gives rise to a realization in a Hilbert space $\mathcal{H}$. A coupling procedure then leads to a symmetric operator in the product space $\mathcal{H} \times \mathcal{K}$ and to the realization of the given Nevanlinna function.
\end{abstract}

\section{INTRODUCTION}

Let $A$ be a closed (not necessarily densely defined) symmetric operator with equal finite deficiency indices $\operatorname{dim}\left(\operatorname{ker}\left(A^{*} \mp i\right)\right)=n<\infty$ in a Hilbert space $\mathcal{H}$ and let $A_{0}$ be a selfadjoint extension of $A$ in $\mathcal{H}$. Recall that the $Q$-function corresponding to the pair $\left\{A, A_{0}\right\}$ is an $n \times n$-matrix function which is determined up to a selfadjoint $n \times n$-matrix by

$$
\frac{Q(\lambda)-Q(\mu)^{*}}{\lambda-\bar{\mu}}=\gamma(\mu)^{*} \gamma(\lambda), \quad \lambda, \mu \in \rho\left(A_{0}\right),
$$

where $\gamma(\lambda)=\left(1+(\lambda-i)\left(A_{0}-\lambda\right)^{-1}\right) \gamma(i)$ and $\gamma(i)$ is an isomorphism from $\mathbb{C}^{n}$ onto the defect subspace $\operatorname{ker}\left(A^{*}-i\right)$; cf. [20, 21, 24]. It follows that $Q$ is a Nevanlinna function; that is, $Q$ holomorphic on $\mathbb{C} \backslash \mathbb{R}, Q(\bar{\lambda})=Q(\lambda)^{*}$ holds for all $\lambda \in \mathbb{C} \backslash \mathbb{R}$ and the selfadjoint matrix $\operatorname{Im} Q(\lambda)$ is nonnegative (nonpositive) for all $\lambda \in \mathbb{C}^{+}\left(\lambda \in \mathbb{C}^{-}\right.$, respectively). Furthermore, definition (1.1) implies $\operatorname{ker}(\operatorname{Im} Q(\lambda))=\{0\}$ for $\lambda \in \mathbb{C} \backslash \mathbb{R}$ and therefore $\operatorname{Im} Q(\lambda)$ is even a uniformly positive (uniformly negative) matrix for all $\lambda \in \mathbb{C}^{+}\left(\lambda \in \mathbb{C}^{-}\right.$, respectively). Nevanlinna functions with this additional property are called uniformly strict. Conversely, if $Q$ is a given uniformly strict $n \times n$-matrix Nevanlinna function, then there exists a Hilbert space $\mathcal{H}$, a closed symmetric operator $A$ with deficiency indices $(n, n)$ and a selfadjoint extension $A_{0}$ of $A$ in $\mathcal{H}$ such that $Q$ is the $Q$-function of the pair $\left\{A, A_{0}\right\}$; see [24].

The notion of $Q$-functions coincides with the modern terminology of Weyl functions associated to boundary triples of symmetric operators, so that, in particular, any uniformly strict Nevanlinna function can be realized as the Weyl function of

Received by the editors January 30, 2008, and, in revised form, October 20, 2008.

2000 Mathematics Subject Classification. Primary 47B50, 30E99; Secondary 47B25, 47A56, $47 \mathrm{~A} 48$.

(C)2009 American Mathematical Society 2685 
a boundary triple and vice versa. The concept of boundary triples and their Weyl functions (see Section 2) is an efficient tool for the description of the spectral properties of selfadjoint extensions of symmetric operators; cf., e.g., 8, 9, 16]. Recently the concept of boundary relations and Weyl families was introduced as a generalization of the notion of boundary triples and Weyl functions in [11, 12, 13. The essential advantage of this concept is that all Nevanlinna functions and, more generally, so-called Nevanlinna families, i.e., $\mathbb{R}$-symmetric holomorphic families of maximal dissipative (in $\mathbb{C}^{+}$) linear relations, can be realized as Weyl families of boundary relations.

In this little note we propose another method to realize (not necessarily uniformly strict) matrix-valued Nevanlinna functions as $Q$-functions or Weyl functions. The advantage is that we stay within the framework of (ordinary) boundary triples, but the model space in general will be a Pontryagin space instead of a Hilbert space. Furthermore, the realization is in general not minimal, but with the exception of the point zero the analytic properties of the given Nevanlinna function are still exactly reflected in the spectral properties of the representing selfadjoint operator or relation. The idea is to decompose a given $n \times n$-matrix Nevanlinna function $\tau$ into a constant part and a "smaller" uniformly strict $m \times m$-matrix Nevanlinna function $\widetilde{\tau}, m \in\{0, \ldots, n\}$, so that $\widetilde{\tau}$ can be minimally realized as the Weyl function corresponding to a symmetric operator $A$ with deficiency indices $(m, m)$ in a Hilbert space $\mathcal{H}$ and a suitable boundary triple. Furthermore, a simple construction shows that a selfadjoint $(n-m) \times(n-m)$-matrix can be realized as the Weyl function of a (nondensely defined) symmetric operator $B$ in a $2(n-m)$-dimensional Pontryagin space $\mathcal{K}$ with negative index $n-m$. Then a coupling procedure yields a boundary triple for the operator $A \times B$ in the Pontryagin space $\mathcal{H} \times \mathcal{K}$ such that $\tau$ coincides with the corresponding Weyl function. We point out that the negative index of the Pontryagin space $\mathcal{H} \times \mathcal{K}$ is $\operatorname{dim}(\operatorname{ker}(\operatorname{Im} \tau(i)))=n-m$ and that for the special case of a uniformly strict Nevanlinna function the space $\mathcal{K}$ is trivial, so that our result reduces to the well-known realization results in [9, 24] if $n=m$.

\section{BOUNDARY TRIPLES AND WEYL FUNCTIONS OF SYMMETRIC RELATIONS in Pontryagin SPACES}

Let $(\mathcal{K},[\cdot, \cdot])$ be a Pontryagin space and let $J$ be a corresponding fundamental symmetry. We study linear relations in $\mathcal{K}$, that is, linear subspaces of $\mathcal{K} \times \mathcal{K}$. The elements in a linear relation will be denoted in the form $\hat{f}=\left\{f, f^{\prime}\right\}, f, f^{\prime} \in \mathcal{K}$. For the set of all closed linear relations in $\mathcal{K}$ we write $\widetilde{\mathcal{C}}(\mathcal{K})$. Linear operators in $\mathcal{K}$ are viewed as linear relations via their graphs. The linear space of bounded linear operators defined on a Pontryagin space $\mathcal{K}$ with values in a Pontryagin space $\mathcal{H}$ is denoted by $\mathcal{L}(\mathcal{K}, \mathcal{H})$. If $\mathcal{K}=\mathcal{H}$, we simply write $\mathcal{L}(\mathcal{K})$.

For a linear relation $A$ in the Pontryagin space $\mathcal{K}$ the adjoint relation $A^{+} \in \widetilde{\mathcal{C}}(\mathcal{K})$ is defined by

$$
A^{+}:=\left\{\hat{f}=\left\{f, f^{\prime}\right\} \in \mathcal{K} \times \mathcal{K}:\left[g^{\prime}, f\right]=\left[g, f^{\prime}\right] \text { for all } \hat{g}=\left\{g, g^{\prime}\right\} \in A\right\} .
$$

A linear relation $A$ in $\mathcal{K}$ is said to be symmetric (selfadjoint) if $A \subset A^{+}\left(A=A^{+}\right.$, respectively). We say that a closed symmetric relation $A \in \widetilde{\mathcal{C}}(\mathcal{K})$ is of defect $m \in \mathbb{N}_{0}$ if the deficiency indices $n_{ \pm}(J A)=\operatorname{dim} \operatorname{ker}\left((J A)^{*} \mp i\right)$ of the closed symmetric relation $J A$ in the Hilbert space $(\mathcal{K},[J \cdot, \cdot])$ are both equal to $m$. Here ${ }^{*}$ denotes the adjoint with respect to the Hilbert scalar product $[J \cdot, \cdot]$. 
For the description of the selfadjoint extensions of a closed symmetric operator or relation in a Pontryagin space we use the concept of boundary triples; see [8, 9, 16] and, e.g., [6, 7] for the Pontryagin and Krĕ̌n space cases respectively.

Definition 2.1. Let $A$ be a closed symmetric relation in the Pontryagin space $\mathcal{K}$. A triple $\left\{\mathcal{G}, \Gamma_{0}, \Gamma_{1}\right\}$ is said to be a boundary triple for $A^{+}$if $\mathcal{G}$ is a Hilbert space, $\Gamma_{0}, \Gamma_{1}: A^{+} \rightarrow \mathcal{G}$ are linear mappings such that the abstract Green's identity

$$
\left[f^{\prime}, g\right]-\left[f, g^{\prime}\right]=\left(\Gamma_{1} \hat{f}, \Gamma_{0} \hat{g}\right)-\left(\Gamma_{0} \hat{f}, \Gamma_{1} \hat{g}\right)
$$

holds for all $\hat{f}=\left\{f, f^{\prime}\right\}, \hat{g}=\left\{g, g^{\prime}\right\} \in A^{+}$, and the mapping

$$
\Gamma:=\left(\begin{array}{c}
\Gamma_{0} \\
\Gamma_{1}
\end{array}\right): A^{+} \rightarrow \mathcal{G} \times \mathcal{G}
$$

is surjective.

Let $A \in \widetilde{\mathcal{C}}(\mathcal{K})$ be a closed symmetric relation in the Pontryagin space $\mathcal{K}$. Then a boundary triple $\left\{\mathcal{G}, \Gamma_{0}, \Gamma_{1}\right\}$ for $A^{+}$exists if and only if $A$ admits a selfadjoint extension in $\mathcal{K}$. In this case the defect of $A$ coincides with $\operatorname{dim} \mathcal{G}$. Assume now that $\left\{\mathcal{G}, \Gamma_{0}, \Gamma_{1}\right\}$ is a boundary triple for $A^{+}$. Then $A=\operatorname{ker}(\Gamma)$ and the linear relations $A_{0}:=\operatorname{ker}\left(\Gamma_{0}\right)$ and $A_{1}:=\operatorname{ker}\left(\Gamma_{1}\right)$ are selfadjoint extensions of $A$ in $\mathcal{K}$. If the resolvent set $\rho\left(A_{0}\right)$ of the selfadjoint relation $A_{0}=\operatorname{ker}\left(\Gamma_{0}\right)$ is nonempty, then it is not difficult to see that

$$
A^{+}=A_{0} \widehat{+} \widehat{\mathcal{N}}_{\lambda, A^{+}}, \quad \widehat{\mathcal{N}}_{\lambda, A^{+}}=\left\{\left\{f_{\lambda}, \lambda f_{\lambda}\right\}: f_{\lambda} \in \mathcal{N}_{\lambda, A^{+}}=\operatorname{ker}\left(A^{+}-\lambda\right)\right\}
$$

holds for all $\lambda \in \rho\left(A_{0}\right)$. Here $\widehat{+}$ denotes the direct sum of subspaces. Recall that in the case $\rho\left(A_{0}\right) \neq \emptyset$ the nonreal spectrum of $A_{0}$ consists of finitely many pairs $\left\{\mu_{j}, \bar{\mu}_{j}\right\}, \mu_{j} \in \mathbb{C}^{+}$, of eigenvalues. For further details on the spectral properties of selfadjoint operators and relations in Pontryagin spaces, we refer the reader to [15, 25, 26].

Associated to a boundary triple are the so-called $\gamma$-field and Weyl function. For symmetric operators in Hilbert spaces the following definition can be found in [8, 9], for the Pontryagin and Kreĭn space cases; see [6, 7].

Definition 2.2. Let $A$ be a closed symmetric relation in the Pontryagin space $\mathcal{K}$, let $\left\{\mathcal{G}, \Gamma_{0}, \Gamma_{1}\right\}$ be a boundary triple for $A^{+}$and $A_{0}=\operatorname{ker}\left(\Gamma_{0}\right)$. Assume $\rho\left(A_{0}\right) \neq \emptyset$ and denote the projection in $\mathcal{K} \times \mathcal{K}$ onto the first component by $\pi_{1}$. The $\gamma$-field $\gamma$ and Weyl function $M$ corresponding to $\left\{\mathcal{G}, \Gamma_{0}, \Gamma_{1}\right\}$ are defined by

$$
\gamma(\lambda)=\pi_{1}\left(\Gamma_{0}\left\lceil\widehat{\mathcal{N}}_{\lambda, A^{+}}\right)^{-1} \text { and } \quad M(\lambda)=\Gamma_{1}\left(\Gamma_{0} \uparrow \widehat{\mathcal{N}}_{\lambda, A^{+}}\right)^{-1}, \quad \lambda \in \rho\left(A_{0}\right) .\right.
$$

Again let $A \in \widetilde{\mathcal{C}}(\mathcal{K})$ be symmetric, let $\left\{\mathcal{G}, \Gamma_{0}, \Gamma_{1}\right\}$ be a boundary triple for $A^{+}$ and assume $\rho\left(A_{0}\right) \neq \emptyset$, where $A_{0}=\operatorname{ker}\left(\Gamma_{0}\right)$. Then the $\gamma$-field $\lambda \mapsto \gamma(\lambda) \in \mathcal{L}(\mathcal{G}, \mathcal{K})$ of $\left\{\mathcal{G}, \Gamma_{0}, \Gamma_{1}\right\}$ is holomorphic on $\rho\left(A_{0}\right)$ and the identity

$$
\gamma(\lambda)=\left(I+(\lambda-\mu)\left(A_{0}-\lambda\right)^{-1}\right) \gamma(\mu)
$$

holds for all $\lambda, \mu \in \rho\left(A_{0}\right)$. The Weyl function $\lambda \mapsto M(\lambda) \in \mathcal{L}(\mathcal{G})$ of $\left\{\mathcal{G}, \Gamma_{0}, \Gamma_{1}\right\}$ is holomorphic on $\rho\left(A_{0}\right)$ and the identities

$$
M(\lambda)-M(\mu)^{*}=(\lambda-\bar{\mu}) \gamma(\mu)^{+} \gamma(\lambda)
$$

and

$$
M(\lambda)=\operatorname{Re} M\left(\lambda_{0}\right)+\gamma\left(\lambda_{0}\right)^{+}\left(\left(\lambda-\operatorname{Re} \lambda_{0}\right)+\left(\lambda-\lambda_{0}\right)\left(\lambda-\bar{\lambda}_{0}\right)\left(A_{0}-\lambda\right)^{-1}\right) \gamma\left(\lambda_{0}\right)
$$


hold for all $\lambda, \mu \in \rho\left(A_{0}\right)$ and any fixed $\lambda_{0} \in \rho\left(A_{0}\right)$. The spectral properties of the selfadjoint extensions of $A$ can be described with the help of the Weyl function $M$; cf. [6, 7, 8, 9].

As $A_{0}$ is a selfadjoint relation in a Pontryagin space, it follows that the Weyl function $M$ belongs to the class of generalized Nevanlinna functions introduced by M.G. Kreın and H. Langer; cf. 21, 22, 23. If, for example, the negative index of the Pontryagin space $\mathcal{K}$ is $\kappa, \kappa \in \mathbb{N}_{0}$, then $M$ belongs to some generalized Nevanlinna class $N_{\tilde{\kappa}}(\mathcal{L}(\mathcal{G})), \tilde{\kappa} \in \mathbb{N}_{0}, \tilde{\kappa} \leq \kappa$; i.e., $M$ is piecewise meromorphic in $\mathbb{C} \backslash \mathbb{R}$, symmetric with respect to the real line $\left(M(\bar{\lambda})=M(\lambda)^{*}\right.$ for all $\lambda$ in the set $\mathfrak{h}(M)$ of points of holomorphy of $M)$, and the kernel

$$
K_{M}(\lambda, \mu):=\frac{M(\lambda)-M(\mu)^{*}}{\lambda-\bar{\mu}}, \quad \lambda, \mu \in \mathbb{C}^{+} \cap \mathfrak{h}(M),
$$

has $\tilde{\kappa}$ negative squares; that is, for all $m \in \mathbb{N}, \lambda_{1}, \ldots, \lambda_{m} \in \mathbb{C}^{+} \cap \mathfrak{h}(M)$ and all $x_{1}, \ldots, x_{m} \in \mathcal{G}$ the selfadjoint matrix

$$
\left(\left(K_{M}\left(\lambda_{i}, \lambda_{j}\right) x_{i}, x_{j}\right)\right)_{i, j=1}^{m}
$$

has at most $\tilde{\kappa}$ negative eigenvalues, and $\tilde{\kappa}$ is minimal with this property. We note that $\tilde{\kappa}=\kappa$ if the defect subspaces $\mathcal{N}_{\lambda, A^{+}}=\operatorname{ker}\left(A^{+}-\lambda\right)$ span a dense subset of $\mathcal{K}$,

$$
\mathcal{K}=\operatorname{clsp}\left\{\mathcal{N}_{\lambda, A^{+}}: \lambda \in \rho\left(A_{0}\right)\right\} .
$$

The functions in the class $N_{0}(\mathcal{L}(\mathcal{G}))$ are called Nevanlinna functions. A function $\tau \in N_{0}(\mathcal{L}(\mathcal{G})$ ) is holomorphic on $\mathbb{C} \backslash \mathbb{R}$ and $\operatorname{Im} \tau(\lambda)$ is nonnegative (nonpositive) for all $\lambda \in \mathbb{C}^{+}\left(\lambda \in \mathbb{C}^{-}\right.$, respectively). It is well-known that Nevanlinna functions can equivalently be characterized by integral representations. More precisely, $\tau$ is an $\mathcal{L}(\mathcal{G})$-valued Nevanlinna function if and only if there exist selfadjoint operators $\alpha, \beta \in \mathcal{L}(\mathcal{G}), \beta \geq 0$, and a nondecreasing selfadjoint operator function $t \mapsto \Sigma(t) \in$ $\mathcal{L}(\mathcal{G})$ on $\mathbb{R}$ such that $\int_{\mathbb{R}} \frac{1}{1+t^{2}} d \Sigma(t) \in \mathcal{L}(\mathcal{G})$ and

$$
\tau(\lambda)=\alpha+\lambda \beta+\int_{-\infty}^{\infty}\left(\frac{1}{t-\lambda}-\frac{t}{1+t^{2}}\right) d \Sigma(t)
$$

holds for all $\lambda \in \mathbb{C} \backslash \mathbb{R}$. Note that if $\mathcal{K}$ is a Hilbert space, $A$ is a closed symmetric relation in $\mathcal{K}$, and $\left\{\mathcal{G}, \Gamma_{0}, \Gamma_{1}\right\}$ is a boundary triple for the adjoint relation $A^{*}$, then the corresponding Weyl function $M$ belongs to the class $N_{0}(\mathcal{L}(\mathcal{G}))$ and the identity (2.1) implies

$$
\operatorname{Im} M(\lambda) \gg 0, \quad \lambda \in \mathbb{C}^{+}, \quad \text { and } \quad \operatorname{Im} M(\lambda) \ll 0, \quad \lambda \in \mathbb{C}^{-} .
$$

Nevanlinna functions with this property are called uniformly strict; cf. [12. If $\operatorname{dim} \mathcal{G}<\infty$, then the Nevanlinna function $\tau$ in (2.4) is either uniformly strict or $\operatorname{ker}(\operatorname{Im} \tau(\lambda)), \lambda \in \mathbb{C} \backslash \mathbb{R}$, is nontrivial.

Example 2.3. Let $\alpha_{j}, \beta_{j} \in \mathcal{L}(\mathcal{G}), j=1, \ldots, m$, be selfadjoint operators in the Hilbert space $\mathcal{G}$ and assume $\beta_{j} \geq 0$. Then the function

$$
\mathbb{C} \backslash \mathbb{R} \ni \lambda \mapsto \tau(\lambda):=\alpha_{1}+\lambda \beta_{1}+\sum_{j=2}^{m} \beta_{j}^{1 / 2}\left(\alpha_{j}-\lambda\right)^{-1} \beta_{j}^{1 / 2}
$$

belongs to the class $N_{0}(\mathcal{L}(\mathcal{G}))$ and $\tau$ is uniformly strict if and only if $0 \in \rho\left(\beta_{j}\right)$ for some $j=1, \ldots, m$. Observe also that $\operatorname{ker}(\operatorname{Im} \tau(\lambda))=\bigcap_{j=1}^{m} \operatorname{ker}\left(\beta_{j}\right), \lambda \in \mathbb{C} \backslash \mathbb{R}$, holds. 


\section{Realization of matrix-VAlued Nevanlinna functions AS WEYL FUNCTIONS}

It is well-known that every uniformly strict $\mathcal{L}(\mathcal{G})$-valued Nevanlinna function $\tau$ can be realized as the Weyl function corresponding to a closed symmetric operator $A$ in a Hilbert space and a suitable boundary triple $\left\{\mathcal{G}, \Gamma_{0}, \Gamma_{1}\right\}$ for $A^{*}$; see, e.g., 8, 9, 12 and 24. In the following we will restrict our investigations to the case of matrix-valued Nevanlinna functions.

3.1. Realization of uniformly strict matrix-valued Nevanlinna functions. Let $\tau \in N_{0}\left(\mathcal{L}\left(\mathbb{C}^{n}\right)\right)$ be a matrix-valued Nevanlinna function given by the integral representation (2.4) and assume that $\tau$ is uniformly strict; i.e., $\operatorname{Im} \tau(\lambda)$ is uniformly positive (uniformly negative) for $\lambda \in \mathbb{C}^{+}\left(\lambda \in \mathbb{C}^{-}\right.$, respectively). In this subsection we recall a particular operator model and realization for $\tau$; cf. [9, 27]. Let $L_{\Sigma}^{2}\left(\mathbb{R}, \mathbb{C}^{n}\right)$ be the Hilbert space of $\mathbb{C}^{n}$-valued functions with the property

$$
\|g\|_{L_{\Sigma}^{2}}:=\left(\int_{\mathbb{R}}(d \Sigma(t) g(t), g(t))\right)^{\frac{1}{2}}<\infty
$$

and denote the corresponding scalar product by $(\cdot, \cdot)_{L_{\Sigma}^{2}}$. For details on the space $L_{\Sigma}^{2}\left(\mathbb{R}, \mathbb{C}^{n}\right)$, see, e.g., [5, [16, 27]. Denote by $\mathcal{G}_{\beta}$ the Hilbert space $\left(\operatorname{ran} \beta,(\cdot, \cdot)_{\beta}\right)$, where

$$
(\beta x, \beta y)_{\beta}:=(\beta x, y), \quad x, y \in \mathbb{C}^{n} .
$$

The orthogonal sum of the maximal multiplication operator with the independent variable in $L_{\Sigma}^{2}\left(\mathbb{R}, \mathbb{C}^{n}\right)$ and the "pure" relation in $\mathcal{G}_{\beta}$ is a selfadjoint relation in the Hilbert space $L_{\Sigma}^{2}\left(\mathbb{R}, \mathbb{C}^{n}\right) \oplus \mathcal{G}_{\beta}$ and will be denoted by $A_{0}$,

$$
A_{0}=\left\{\{f(t) \oplus 0, t f(t) \oplus \tilde{b}\}: f(t), t f(t) \in L_{\Sigma}^{2}\left(\mathbb{R}, \mathbb{C}^{n}\right), \tilde{b} \in \mathcal{G}_{\beta}\right\} .
$$

For brevity we have denoted here the functions $t \mapsto f(t)$ and $t \mapsto t f(t)$ in $L_{\Sigma}^{2}\left(\mathbb{R}, \mathbb{C}^{n}\right)$ just by $f(t)$ and $t f(t)$ and we agree to use this notation also in the next theorem. The following result was proved by V.A. Derkach and M.M. Malamud in [9].

Theorem 3.1. Let $\tau \in N_{0}\left(\mathcal{L}\left(\mathbb{C}^{n}\right)\right)$ be a uniformly strict matrix-valued Nevanlinna function of the form (2.4). Let $L_{\Sigma}^{2}\left(\mathbb{R}, \mathbb{C}^{n}\right) \oplus \mathcal{G}_{\beta}$ be the Hilbert space from above and let $A_{0}$ be the selfadjoint relation from (3.1). Then

$$
A=\left\{\{f(t) \oplus 0, t f(t) \oplus \tilde{b}\} \in A_{0}: \int_{\mathbb{R}} d \Sigma(t) f(t)+\tilde{b}=0\right\}
$$

is a closed simple symmetric operator in $L_{\Sigma}^{2}\left(\mathbb{R}, \mathbb{C}^{n}\right) \oplus \mathcal{G}_{\beta}$; the adjoint is

$$
\begin{aligned}
A^{*}=\{\{f(t) \oplus b, \tilde{f}(t) \oplus \tilde{b}\}: & f(t), \tilde{f}(t) \in L_{\Sigma}^{2}\left(\mathbb{R}, \mathbb{C}^{n}\right), \tilde{b} \in \mathcal{G}_{\beta}, \\
& \left.\exists h \in \mathbb{C}^{n}: \tilde{f}(t)-t f(t)=-h, b=\beta h\right\} ;
\end{aligned}
$$

and $\left\{\mathbb{C}^{n}, \Gamma_{0}, \Gamma_{1}\right\}$, where

$$
\Gamma_{0} \hat{f}:=h, \quad \Gamma_{1} \hat{f}:=\tilde{b}+\alpha h+\int_{\mathbb{R}} d \Sigma(t) \frac{t \tilde{f}(t)+f(t)}{1+t^{2}},
$$

$\hat{f}=\{f(t) \oplus b, \tilde{f}(t) \oplus \tilde{b}\} \in A^{*}$, is a boundary triple for $A^{*}$ with $A_{0}=\operatorname{ker}\left(\Gamma_{0}\right)$ and the corresponding Weyl function coincides with $\tau$. 
3.2. Realization of nonstrict matrix-valued Nevanlinna functions. The following theorem is the main result of this paper. We prove that every (in general nonstrict) matrix-valued Nevanlinna function $\tau$ can be realized as the Weyl function corresponding to a symmetric operator in a Pontryagin space and a suitable boundary triplet for its adjoint. We point out that the realization constructed here is not minimal if $\tau$ is nonstrict, so that the analytic properties of $\tau$ are in general not completely reflected in the spectral properties of the representing selfadjoint operator or relation; see Remark 3.3.

Theorem 3.2. Let $\tau \in N_{0}\left(\mathcal{L}\left(\mathbb{C}^{n}\right)\right)$ and let $\kappa=\operatorname{dim}(\operatorname{ker}(\operatorname{Im} \tau(i)))$. Then there exists a Pontryagin space $\widetilde{\mathcal{K}}$ with negative index $\kappa$, a closed symmetric operator $S$ in $\widetilde{\mathcal{K}}$ and a boundary triple $\left\{\mathbb{C}^{n}, \widetilde{\Gamma}_{0}, \widetilde{\Gamma}_{1}\right\}$ for $S^{+}$such that the corresponding Weyl function coincides with $\tau$.

Proof. The proof of Theorem 3.2 consists of three steps. In the first step the Nevanlinna function $\tau$ is written as the sum of a "smaller" uniformly strict Nevanlinna function $\tau_{11}$ and a selfadjoint constant,

$$
\tau(\lambda)=\left(\begin{array}{cc}
\tau_{11}(\lambda) & 0 \\
0 & 0
\end{array}\right)+\left(\begin{array}{cc}
0 & \alpha_{12} \\
\alpha_{12}^{*} & \alpha_{22}
\end{array}\right)
$$

in the second step the constant $\alpha_{22}$ is realized as the Weyl function corresponding to a symmetric operator and a suitable boundary triple in a Pontryagin space; and in the last step it is shown that the realization of $\tau_{11}$ together with the realization of $\alpha_{22}$ yields a boundary triple for the orthogonal sum of the corresponding symmetric operators such that $\tau$ becomes the associated Weyl function.

Step 1. Let us regard the space $\mathbb{C}^{n}$ as the direct orthogonal sum of the subspaces $\operatorname{ran}(\operatorname{Im} \tau(i))$ and $\operatorname{ker}(\operatorname{Im} \tau(i))$,

$$
\mathbb{C}^{n}=\operatorname{ran}(\operatorname{Im} \tau(i)) \oplus \operatorname{ker}(\operatorname{Im} \tau(i)),
$$

and decompose the function $\tau$ accordingly, i.e.,

$$
\tau(\lambda)=\left(\begin{array}{ll}
\tau_{11}(\lambda) & \tau_{12}(\lambda) \\
\tau_{21}(\lambda) & \tau_{22}(\lambda)
\end{array}\right):\left(\begin{array}{c}
\operatorname{ran}(\operatorname{Im} \tau(i)) \\
\operatorname{ker}(\operatorname{Im} \tau(i))
\end{array}\right) \rightarrow\left(\begin{array}{l}
\operatorname{ran}(\operatorname{Im} \tau(i)) \\
\operatorname{ker}(\operatorname{Im} \tau(i))
\end{array}\right) .
$$

Let $\kappa=\operatorname{dim}(\operatorname{ker}(\operatorname{Im} \tau(i))), \kappa \in 0, \ldots, n$, denote the canonical embedding of $\operatorname{ran}(\operatorname{Im} \tau(i))$ in $\mathbb{C}^{n}$ by $\iota$ and let $\pi$ be the orthogonal projection from $\mathbb{C}^{n}$ onto $\operatorname{ran}(\operatorname{Im} \tau(i))$ so that $\iota^{*}=\pi$.

As a consequence of the integral representation (2.4) we have

$$
\operatorname{Im} \tau(i)=\beta+\int_{-\infty}^{\infty} \frac{1}{1+t^{2}} d \Sigma(t)
$$

and since $\beta \geq 0$ and $t \mapsto \Sigma(t)$ is a nondecreasing selfadjoint matrix function we conclude that every $x \in \operatorname{ker}(\operatorname{Im} \tau(i))$ belongs to $\operatorname{ker}(\beta)$ and the $\mathbb{C}^{n}$-valued function $t \mapsto \Sigma(t) x, x \in \operatorname{ker}(\operatorname{Im} \tau(i))$, is equal to a constant. Therefore, if we decompose the selfadjoint matrices $\alpha, \beta$ in (2.4) with respect to the space decomposition (3.2),

$$
\alpha=\left(\begin{array}{ll}
\alpha_{11} & \alpha_{12} \\
\alpha_{12}^{*} & \alpha_{22}
\end{array}\right), \quad \beta=\left(\begin{array}{cc}
\beta_{11} & 0 \\
0 & 0
\end{array}\right)
$$

it follows that $\tau_{12}(\lambda)=\alpha_{12}$ and $\tau_{22}(\lambda)=\alpha_{22}$, and the symmetry property $\tau(\bar{\lambda})=$ $\tau(\lambda)^{*}$ implies

$$
\tau(\lambda)=\left(\begin{array}{cc}
\tau_{11}(\lambda) & \alpha_{12} \\
\alpha_{12}^{*} & \alpha_{22}
\end{array}\right):\left(\begin{array}{l}
\operatorname{ran}(\operatorname{Im} \tau(i)) \\
\operatorname{ker}(\operatorname{Im} \tau(i))
\end{array}\right) \rightarrow\left(\begin{array}{c}
\operatorname{ran}(\operatorname{Im} \tau(i)) \\
\operatorname{ker}(\operatorname{Im} \tau(i))
\end{array}\right) .
$$


It is clear that $\tau_{11}$ is a Nevanlinna function with the integral representation

$$
\tau_{11}(\lambda)=\alpha_{11}+\lambda \beta_{11}+\int_{-\infty}^{\infty}\left(\frac{1}{t-\lambda}-\frac{t}{1+t^{2}}\right) d \Sigma_{11}(t),
$$

where $t \mapsto \Sigma_{11}(t):=\pi \Sigma(t) \iota \in \mathcal{L}(\operatorname{ran}(\operatorname{Im} \tau(i)))$ is a nondecreasing selfadjoint matrix function on $\mathbb{R}$ such that $\int_{\mathbb{R}} \frac{1}{1+t^{2}} d \Sigma_{11}(t) \in \mathcal{L}(\operatorname{ran}(\operatorname{Im} \tau(i)))$. Moreover,

$$
\operatorname{Im} \tau_{11}(\lambda) \gg 0, \quad \lambda \in \mathbb{C}^{+}, \quad \text { and } \operatorname{Im} \tau_{11}(\lambda) \ll 0, \quad \lambda \in \mathbb{C}^{-} ;
$$

that is, $\tau_{11}$ is uniformly strict. The decomposition (3.3) of a nonstrict matrix Nevanlinna function can also be found in [17.

Step 2. We equip the Hilbert space $\operatorname{ker}(\operatorname{Im} \tau(i)) \oplus \operatorname{ker}(\operatorname{Im} \tau(i))$ with the indefinite inner product $[\cdot, \cdot]:=(J \cdot, \cdot)$, where $J=\left(\begin{array}{cc}0 & I \\ I & 0\end{array}\right)$ and $(\cdot, \cdot)$ is the Hilbert scalar product on $\operatorname{ker}(\operatorname{Im} \tau(i)) \oplus \operatorname{ker}(\operatorname{Im} \tau(i))$. Then

$$
\mathcal{K}=(\operatorname{ker}(\operatorname{Im} \tau(i)) \oplus \operatorname{ker}(\operatorname{Im} \tau(i)),[\cdot, \cdot])
$$

is a Pontryagin space with negative index $\kappa=\operatorname{dim}(\operatorname{ker}(\operatorname{Im} \tau(i)))$ and the matrix

$$
B_{0}:=\left(\begin{array}{cc}
0 & I \\
0 & 0
\end{array}\right) \in \mathcal{L}(\operatorname{ker}(\operatorname{Im} \tau(i)) \oplus \operatorname{ker}(\operatorname{Im} \tau(i)))
$$

is selfadjoint in $\mathcal{K}$. For every $\lambda \in \mathbb{C} \backslash\{0\}$ we have

$$
\left(B_{0}-\lambda\right)^{-1}=\left(\begin{array}{cc}
-\lambda^{-1} & -\lambda^{-2} \\
0 & -\lambda^{-1}
\end{array}\right) \in \mathcal{L}(\mathcal{K}) .
$$

Let $\lambda_{0} \in \mathbb{C} \backslash\{0\}, \gamma_{\lambda_{0}}:=(I, 0)^{\top}$ and define for $\lambda \in \mathbb{C} \backslash\{0\}$,

$$
\begin{aligned}
\gamma(\lambda): \operatorname{ker}(\operatorname{Im} \tau(i)) & \rightarrow \mathcal{K}, \\
x & \mapsto\left(I+\left(\lambda-\lambda_{0}\right)\left(B_{0}-\lambda\right)^{-1}\right) \gamma_{\lambda_{0}} x=\left(\frac{\lambda_{0}}{\lambda} x, 0\right)^{\top} .
\end{aligned}
$$

Then obviously $\operatorname{ran} \gamma(\lambda)=\operatorname{ker}(\operatorname{Im} \tau(i)) \times\{0\}$. From

$$
\gamma(\eta)^{+}: \mathcal{K} \rightarrow \operatorname{ker}(\operatorname{Im} \tau(i)), \quad(x, y)^{\top} \mapsto \frac{\bar{\lambda}_{0}}{\bar{\eta}} y, \quad \eta \in \mathbb{C} \backslash\{0\},
$$

we obtain $\gamma(\eta)^{+} \gamma(\lambda)=0$ for all $\lambda, \eta \in \mathbb{C} \backslash\{0\}$. Consider the closed symmetric operator

$$
B:=B_{0} \uparrow(\operatorname{ker}(\operatorname{Im} \tau(i)) \times\{0\})
$$

in $\mathcal{K}$. Then we have $\mathcal{N}_{\lambda, B^{+}}=\operatorname{ran} \gamma(\lambda)$ for all $\lambda \in \mathbb{C} \backslash\{0\}$, the defect of $B$ coincides with $\kappa=\operatorname{dim}(\operatorname{ker}(\operatorname{Im} \tau(i)))$, and $\mathcal{N}_{\lambda, B^{+}}[\perp] \mathcal{N}_{\eta, B^{+}}$holds for all $\lambda, \eta \in \mathbb{C} \backslash\{0\}$. For a fixed $\mu \in \mathbb{C} \backslash\{0\}$ we write the elements $\hat{g} \in B^{+}=B_{0} \widehat{+}_{\mathcal{N}_{\mu, B^{+}}}$in the form

$$
\hat{g}=\left\{g_{0}, B_{0} g_{0}\right\}+\{\gamma(\mu) x, \mu \gamma(\mu) x\}, \quad g_{0} \in \mathcal{K}, \quad x \in \operatorname{ker}(\operatorname{Im} \tau(i)) .
$$

We leave it to the reader to check that the triple $\left\{\operatorname{ker}(\operatorname{Im} \tau(i)), \Gamma_{0}^{\prime}, \Gamma_{1}^{\prime}\right\}$, where

$$
\Gamma_{0}^{\prime} \hat{g}:=x \quad \text { and } \quad \Gamma_{1}^{\prime} \hat{g}:=\gamma(\mu)^{+}\left(B_{0}-\bar{\mu}\right) g_{0}+\alpha_{22} x,
$$

is a boundary triple for $B^{+}$and the corresponding Weyl function is $\alpha_{22}$.

Step 3. Since $\tau_{11}$ is an $\mathcal{L}(\operatorname{ran}(\operatorname{Im} \tau(i)))$-valued uniformly strict Nevanlinna function there exists a Hilbert space $\mathcal{H}$, a closed symmetric operator $A$ in $\mathcal{H}$ and a boundary triple $\left\{\operatorname{ran}(\operatorname{Im} \tau(i)), \Gamma_{0}, \Gamma_{1}\right\}$ for $A^{*}$ such that $\tau_{11}$ is the corresponding Weyl function; cf. Theorem 3.1 .

In the following we consider the closed symmetric operator $S:=A \times B$ in the Pontryagin space $\widetilde{\mathcal{K}}:=\mathcal{H} \times \mathcal{K}$ and its adjoint $S^{+}=A^{*} \times B^{+}$. The elements in 
$A^{*} \times B^{+}$will be denoted in the form $\left\{\{f, g\},\left\{f^{\prime}, g^{\prime}\right\}\right\}$, where $\hat{f}=\left\{f, f^{\prime}\right\} \in A^{*}$ and $\hat{g}=\left\{g, g^{\prime}\right\} \in B^{+}$. We claim that $\left\{\mathbb{C}^{n}, \widetilde{\Gamma}_{0}, \widetilde{\Gamma}_{1}\right\}$, where

$$
\widetilde{\Gamma}_{0}\{\hat{f}, \hat{g}\}:=\left(\begin{array}{c}
\Gamma_{0} \hat{f} \\
\Gamma_{0}^{\prime} \hat{g}
\end{array}\right) \text { and } \widetilde{\Gamma}_{1}\{\hat{f}, \hat{g}\}:=\left(\begin{array}{c}
\Gamma_{1} \hat{f}+\alpha_{12} \Gamma_{0}^{\prime} \hat{g} \\
\Gamma_{1}^{\prime} \hat{g}+\alpha_{12}^{*} \Gamma_{0} \hat{f},
\end{array}\right), \quad\{\hat{f}, \hat{g}\} \in A^{*} \times B^{+},
$$

is a boundary triple for $S^{+}$such that the corresponding Weyl function coincides with $\tau$. This can also be deduced from [10, §3.3]. However, for completeness we give a short direct proof. Indeed, for $\{\hat{f}, \hat{g}\},\{\hat{h}, \hat{k}\} \in A^{*} \times B^{+}$,

$$
\begin{aligned}
& \left(\widetilde{\Gamma}_{1}\{\hat{f}, \hat{g}\}, \widetilde{\Gamma}_{0}\{\hat{h}, \hat{k}\}\right)_{\mathbb{C}^{n}}-\left(\widetilde{\Gamma}_{0}\{\hat{f}, \hat{g}\}, \widetilde{\Gamma}_{1}\{\hat{h}, \hat{k}\}\right)_{\mathbb{C}^{n}} \\
& =\left(\left(\begin{array}{l}
\Gamma_{1} \hat{f}+\alpha_{12} \Gamma_{0}^{\prime} \hat{g} \\
\Gamma_{1}^{\prime} \hat{g}+\alpha_{12}^{*} \Gamma_{0} \hat{f}
\end{array}\right),\left(\begin{array}{c}
\Gamma_{0} \hat{h} \\
\Gamma_{0}^{\prime} \hat{k}
\end{array}\right)\right)_{\mathbb{C}^{n}}-\left(\left(\begin{array}{c}
\Gamma_{0} \hat{f} \\
\Gamma_{0}^{\prime} \hat{g}
\end{array}\right),\left(\begin{array}{l}
\Gamma_{1} \hat{h}+\alpha_{12} \Gamma_{0}^{\prime} \hat{k} \\
\Gamma_{1}^{\prime} \hat{k}+\alpha_{12}^{*} \Gamma_{0} \hat{h}
\end{array}\right)\right)_{\mathbb{C}^{n}}
\end{aligned}
$$

holds. Since $\left\{\operatorname{ran}(\operatorname{Im} \tau(i)), \Gamma_{0}, \Gamma_{1}\right\}$ and $\left\{\operatorname{ker}(\operatorname{Im} \tau(i)), \Gamma_{0}^{\prime}, \Gamma_{1}^{\prime}\right\}$ are boundary triples for $A^{*}$ and $B^{+}$, respectively, we conclude that the above expression becomes

$$
\begin{aligned}
&\left(\Gamma_{1} \hat{f}, \Gamma_{0} \hat{h}\right)_{\operatorname{ran}(\operatorname{Im} \tau(i))}-\left(\Gamma_{0} \hat{f}, \Gamma_{1} \hat{h}\right)_{\operatorname{ran}(\operatorname{Im} \tau(i))} \\
&+\left(\Gamma_{1}^{\prime} \hat{g}, \Gamma_{0}^{\prime} \hat{k}\right)_{\operatorname{ker}(\operatorname{Im} \tau(i))}-\left(\Gamma_{0}^{\prime} \hat{g}, \Gamma_{1}^{\prime} \hat{k}\right)_{\operatorname{ker}(\operatorname{Im} \tau(i))} \\
&=\left(f^{\prime}, h\right)_{\mathcal{H}}-\left(f, h^{\prime}\right)_{\mathcal{H}}+\left[g^{\prime}, k\right]_{\mathcal{K}}-\left[g, k^{\prime}\right]_{\mathcal{K}} \\
&=\left[\left\{f^{\prime}, g^{\prime}\right\},\{h, k\}\right]_{\widetilde{\mathcal{K}}}-\left[\{f, g\},\left\{h^{\prime}, k^{\prime}\right\}\right]_{\tilde{\mathcal{K}}} .
\end{aligned}
$$

Therefore $\left\{\mathbb{C}^{n}, \widetilde{\Gamma}_{0}, \widetilde{\Gamma}_{1}\right\}$ satisfies the abstract Green's identity. The surjectivity of the mapping $\left(\widetilde{\Gamma}_{0}, \widetilde{\Gamma}_{1}\right)^{\top}: S^{+} \rightarrow \mathbb{C}^{n} \times \mathbb{C}^{n}$ follows easily from the surjectivity of the mappings

$$
\begin{gathered}
\left(\begin{array}{l}
\Gamma_{0} \\
\Gamma_{1}
\end{array}\right): A^{*} \rightarrow \operatorname{ran}(\operatorname{Im} \tau(i)) \times \operatorname{ran}(\operatorname{Im} \tau(i)), \\
\left(\begin{array}{c}
\Gamma_{0}^{\prime} \\
\Gamma_{1}^{\prime}
\end{array}\right): B^{+} \rightarrow \operatorname{ker}(\operatorname{Im} \tau(i)) \times \operatorname{ker}(\operatorname{Im} \tau(i)) .
\end{gathered}
$$

Hence $\left\{\mathbb{C}^{n}, \widetilde{\Gamma}_{0}, \widetilde{\Gamma}_{1}\right\}$ is a boundary triple for $S^{+}$. It remains to show that the corresponding Weyl function coincides with $\tau$. For this, note that

$$
\widehat{\mathcal{N}}_{\lambda, S^{+}}=\widehat{\mathcal{N}}_{\lambda, A^{*} \times B^{+}}=\widehat{\mathcal{N}}_{\lambda, A^{*}} \times \widehat{\mathcal{N}}_{\lambda, B^{+}}, \quad \lambda \in \mathbb{C} \backslash \mathbb{R},
$$

and let $\left\{\left\{f_{\lambda}, g_{\lambda}\right\},\left\{\lambda f_{\lambda}, \lambda g_{\lambda}\right\}\right\} \in S^{+}=A^{*} \times B^{+}$, where $\left\{f_{\lambda}, \lambda f_{\lambda}\right\} \in \widehat{\mathcal{N}}_{\lambda, A^{*}}$ and $\left\{g_{\lambda}, \lambda g_{\lambda}\right\} \in \widehat{\mathcal{N}}_{\lambda, B^{+}}$. Since

$$
\tau_{11}(\lambda) \Gamma_{0} \hat{f}_{\lambda}=\Gamma_{1} \hat{f}_{\lambda} \quad \text { and } \quad \alpha_{22} \Gamma_{0}^{\prime} \hat{g}_{\lambda}=\Gamma_{1}^{\prime} \hat{g}_{\lambda}, \quad \lambda \in \mathbb{C} \backslash \mathbb{R},
$$

we conclude

$$
\tau(\lambda) \widetilde{\Gamma}_{0}\left\{\hat{f}_{\lambda}, \hat{g}_{\lambda}\right\}=\left(\begin{array}{cc}
\tau_{11}(\lambda) & \alpha_{12} \\
\alpha_{12}^{*} & \alpha_{22}
\end{array}\right)\left(\begin{array}{c}
\Gamma_{0} \hat{f}_{\lambda} \\
\Gamma_{0}^{\prime} \hat{g}_{\lambda}
\end{array}\right)=\left(\begin{array}{c}
\Gamma_{1} \hat{f}_{\lambda}+\alpha_{12} \Gamma_{0}^{\prime} \hat{g}_{\lambda} \\
\alpha_{12}^{*} \Gamma_{0} \hat{f}_{\lambda}+\Gamma_{1}^{\prime} \hat{g}_{\lambda}
\end{array}\right)=\widetilde{\Gamma}_{1}\left\{\hat{f}_{\lambda}, \hat{g}_{\lambda}\right\}
$$

for all $\lambda \in \mathbb{C} \backslash \mathbb{R}$; that is, $\tau$ is the Weyl function of $\left\{\mathbb{C}^{n}, \widetilde{\Gamma}_{0}, \widetilde{\Gamma}_{1}\right\}$.

Remark 3.3. The defect subspaces $\mathcal{N}_{\lambda, B^{+}}=\operatorname{ker}\left(B^{+}-\lambda\right), \lambda \in \mathbb{C} \backslash \mathbb{R}$, coincide with the closed subspace $\operatorname{ker}(\operatorname{Im}(\tau(i))) \times\{0\}$ of $\mathcal{K}$ and hence

$$
\mathcal{K} \neq \operatorname{clsp}\left\{\mathcal{N}_{\lambda, B^{+}}: \lambda \in \mathbb{C} \backslash \mathbb{R}\right\}=\operatorname{ker}(\operatorname{Im} \tau(i)) \times\{0\} .
$$


Therefore the representation of the function $\tau$ in Theorem 3.2 is not minimal if $\kappa=\operatorname{dim}(\operatorname{ker}(\operatorname{Im} \tau(i)))>0$,

$$
\widetilde{\mathcal{K}} \neq \operatorname{clsp}\left\{\mathcal{N}_{\lambda, S^{+}}: \lambda \in \mathbb{C} \backslash \mathbb{R}\right\}=\operatorname{clsp}\left\{\mathcal{N}_{\lambda, A^{*}} \times \mathcal{N}_{\lambda, B^{+}}: \lambda \in \mathbb{C} \backslash \mathbb{R}\right\} .
$$

This implies that the analytic properties of $\tau$ are in general not completely reflected by the spectral properties of the selfadjoint operator or relation $S_{0}:=\operatorname{ker}\left(\widetilde{\Gamma}_{0}\right)$ in $\widetilde{\mathcal{K}}$. If the representation of $\tau_{11}$ is chosen to be minimal, then in the realization constructed above this disadvantage does only arise at the point zero. More precisely, here zero is an eigenvalue of $S_{0}$ although the measure $d \Sigma$ in the integral representation (2.4) of $\tau$ does not necessarily have a point mass at zero. Nevertheless, the behaviour of $d \Sigma$ or $\tau$ in $\mathbb{R} \backslash\{0\}$ or $\mathbb{C} \backslash\{0\}$ is exactly reflected in the nonzero spectrum of $S_{0}$. In particular, if $\mathfrak{h}(\tau)$ denotes the domain of holomorphy of $\tau$, then $\mathfrak{h}(\tau) \backslash\{0\}=\rho\left(S_{0}\right) \backslash\{0\}$.

Remark 3.4. The Pontryagin space $\widetilde{\mathcal{K}}$ in Theorem 3.2 can be chosen as

$$
\left(L_{\Sigma_{11}}^{2}(\mathbb{R}, \operatorname{ran}(\operatorname{Im} \tau(i))) \oplus \mathcal{G}_{\beta_{11}}\right) \times(\operatorname{ker}(\operatorname{Im} \tau(i)))^{2}
$$

equipped with the inner product

$$
\begin{aligned}
& {\left[\left(f \oplus \beta_{11} x\right) \times\left(g \oplus g^{\prime}\right),\left(\tilde{f} \oplus \beta_{11} y\right) \times\left(h \oplus h^{\prime}\right)\right]} \\
& :=\int_{\mathbb{R}}\left(d \Sigma_{11}(t) f(t), \tilde{f}(t)\right)_{\operatorname{ran}(\operatorname{Im} \tau(i))}+\left(\beta_{11} x, y\right)_{\operatorname{ran}(\operatorname{Im} \tau(i))} \\
& +\left(g^{\prime}, h\right)_{\operatorname{ker}(\operatorname{Im} \tau(i))}+\left(g, h^{\prime}\right)_{\operatorname{ker}(\operatorname{Im} \tau(i))},
\end{aligned}
$$

with $f, \tilde{f} \in L_{\Sigma_{11}}^{2}(\mathbb{R}, \operatorname{ran}(\operatorname{Im} \tau(i))), x, y \in \operatorname{ran}(\operatorname{Im} \tau(i))$ and $g, g^{\prime}, h, h^{\prime} \in \operatorname{ker}(\operatorname{Im} \tau(i))$. Then the symmetric operator $S$ is the direct product of the operator $A$ in Theorem 3.1 (where $\Sigma$ and $\beta$ have to be replaced by $\Sigma_{11}$ and $\beta_{11}$ ) and the nondensely defined operator $B$ in (3.4).

Remark 3.5. Realizations of matrix and operator-valued (generalized) Nevanlinna functions of the form (2.2) satisfying the minimality condition (2.3) can be found in 14, 18, 19, 24, but a nonstrict Nevanlinna function cannot be interpreted as a usual Weyl function or $Q$-function. In the recent papers [11, 12, 13, by V.A. Derkach, S. Hassi, M.M. Malamud and H.S.V. de Snoo the concept of boundary triples and Weyl functions was generalized to so-called boundary relations (multivalued boundary mappings) and Weyl families. In this framework also nonstrict Nevanlinna functions, and, more generally, Nevanlinna families, can be minimally realized as Weyl families associated to boundary relations of symmetric operators in Hilbert spaces; see also [3]. For the case of a nonstrict Nevanlinna function that is not equal to a constant, the corresponding boundary relation is necessarily multivalued.

\section{An application: Boundary VAlue PROBlems WITH $\lambda$-DEPENDENT BOUNDARY CONDITIONS}

Boundary value problems in which the spectral parameter appears nonlinearly in the boundary condition have been studied in many different frameworks in recent decades; see, e.g., 1, 2, 4, 6, 7, 10, 13, 14, and the references therein. We consider a boundary value problem with a nonstrict matrix Nevanlinna function $\tau$ in the boundary condition and apply Theorem 3.2 to construct a linearization in a straightforward way. 
Let $T$ be a densely defined closed symmetric operator with equal finite deficiency indices $\operatorname{dim}\left(\operatorname{ker}\left(T^{*} \mp i\right)\right)=n<\infty$ in a Hilbert space $\widetilde{\mathcal{H}}$ and let $\left\{\mathbb{C}^{n}, \Upsilon_{0}, \Upsilon_{1}\right\}$ be a boundary triple for the operator $T^{*}$ with corresponding Weyl function $M$. The selfadjoint extension $\operatorname{ker}\left(\Upsilon_{0}\right)$ will be denoted by $T_{0}$. Let $\tau \in N_{0}\left(\mathcal{L}\left(\mathbb{C}^{n}\right)\right)$ be a (in general nonstrict) Nevanlinna function with domain of holomorphy $\mathfrak{h}(\tau)$. We consider the following abstract $\lambda$-dependent boundary value problem: For a given $k \in \widetilde{\mathcal{H}}$ and $\lambda \in \mathfrak{h}(\tau)$ find $h \in \operatorname{dom} T^{*}$ such that

$$
\left(T^{*}-\lambda\right) h=k \quad \text { and } \quad \tau(\lambda) \Upsilon_{0} \hat{h}+\Upsilon_{1} \hat{h}=0, \quad \hat{h}=\left\{h, T^{*} h\right\}
$$

holds. According to Theorem 3.2 and Remark 3.3 there exist a Pontryagin space $\widetilde{\mathcal{K}}$, a closed symmetric operator $S$ in $\widetilde{\mathcal{K}}$ and a boundary triple $\left\{\mathbb{C}^{n}, \widetilde{\Gamma}_{0}, \widetilde{\Gamma}_{1}\right\}$ such that $\tau$ is the corresponding Weyl function and for the selfadjoint relation $S_{0}=\operatorname{ker}\left(\widetilde{\Gamma}_{0}\right)$ we have $\rho\left(S_{0}\right) \backslash\{0\}=\mathfrak{h}(\tau) \backslash\{0\}$. With the help of $S$ and $\left\{\mathbb{C}^{n}, \widetilde{\Gamma}_{0}, \widetilde{\Gamma}_{1}\right\}$ a selfadjoint solution operator $\widetilde{A} \subset T^{*} \times S^{+}$for the problem (4.1) in the Pontryagin space $\tilde{\mathcal{H}} \times \widetilde{\mathcal{K}}$ can be constructed. For the special case of a uniformly strict Nevanlinna function $\tau$, the explicit form of $\widetilde{A}$ in the following theorem is known, $\widetilde{\mathcal{K}}$ is a Hilbert space, and it is not necessary to exclude the point $\lambda=0$; see Remark 4.2 ,

Theorem 4.1. The operator $\widetilde{A}\left(\begin{array}{c}h \\ k\end{array}\right)=\left(\begin{array}{c}T^{*} h \\ k^{\prime}\end{array}\right)$ defined on

$$
\operatorname{dom} \widetilde{A}=\left\{\left(\begin{array}{l}
h \\
k
\end{array}\right) \in \widetilde{\mathcal{H}} \times \widetilde{\mathcal{K}}:\left(\begin{array}{c}
\Upsilon_{0} \hat{h}+\widetilde{\Gamma}_{0} \hat{k}=0 \\
\Upsilon_{1} \hat{h}-\widetilde{\Gamma}_{1} \hat{k}=0
\end{array}\right) \text { for some } \hat{k}=\left\{k, k^{\prime}\right\} \in S^{+}\right\}
$$

is a selfadjoint extension of $T$ in the Pontryagin space $\widetilde{\mathcal{H}} \times \widetilde{\mathcal{K}}$ with $\sigma(\widetilde{A}) \subset \mathbb{R}$. For $\lambda \in \rho(\widetilde{A}) \cap \mathfrak{h}(\tau) \cap \rho\left(T_{0}\right), \lambda \neq 0$, the unique solution of (4.1) is given by

$$
h=P_{\widetilde{\mathcal{H}}}(\widetilde{A}-\lambda)^{-1}\left(\begin{array}{l}
k \\
0
\end{array}\right) .
$$

For $\lambda \in \mathfrak{h}(\tau) \backslash\{0\}$ the problem (4.1) with $k=0$ has a nontrivial solution $h \in \widetilde{\mathcal{H}}$ if and only if $\lambda \in \sigma_{p}(\widetilde{A})$. In this case the solutions are given by the components of the eigenvectors of $\widetilde{A}$ in $\widetilde{\mathcal{H}}$.

Proof. Observe that $\widetilde{A}$ is an operator since $h=k=0$ implies $\hat{h}=\left\{h, T^{*} h\right\}=0$ and hence $\widetilde{\Gamma}_{0} \hat{k}=\widetilde{\Gamma}_{1} \hat{k}=0$; i.e., $\hat{k} \in S$ and as $S$ is an operator, $k^{\prime}=0$ follows. It can be shown that $\widetilde{A}$ is selfadjoint and that $\lambda \in \rho\left(T_{0}\right) \cap \rho\left(S_{0}\right)$ belongs to $\rho(\widetilde{A})$ if and only if $\operatorname{ker}(M(\lambda)+\tau(\lambda))=\{0\}$ holds. Therefore, as $\mathbb{C} \backslash \mathbb{R} \subset\left(\rho\left(T_{0}\right) \cap \rho\left(S_{0}\right)\right)$ and the Weyl function $M \in N_{0}\left(\mathcal{L}\left(\mathbb{C}^{n}\right)\right)$ is uniformly strict we obtain $\sigma(\widetilde{A}) \subset \mathbb{R}$. The proof of the remaining assertions is essentially the same as in [4, Theorem 4.1] and [2, Theorem 4.5].

Remark 4.2. Note that here $\sigma(\widetilde{A})$ is real although $\widetilde{A}$ acts in a Pontryagin space and that with the possible exception of the point $\lambda=0$ the boundary value problem (4.1) can be completely solved with the help of the linearization $\widetilde{A}$. We note that there exists also a minimal solution operator of (4.1) acting in a Hilbert space, but for the construction, a transformation of $\tau$ into the orthogonal sum of a strict Nevanlinna function and a purely multivalued relation is necessary (cf. [10, §5.2]), or the concept of boundary relations and Weyl families has to be used; see [13. 


\section{REFERENCES}

[1] T.Ya. Azizov, B. Ćurgus, A. Dijksma: Standard symmetric operators in Pontryagin spaces: A generalized von Neumann formula and minimality of boundary coefficients, J. Funct. Anal. 198 (2003), 361-412. MR1964544 (2004a:47034)

[2] J. Behrndt, Boundary value problems with eigenvalue depending boundary conditions, Math. Nachr., to appear.

[3] J. Behrndt, S. Hassi, H.S.V. de Snoo, Functional models for Nevanlinna families, Opuscula Math. 28 (2008), 233-245. MR2425117

[4] J. Behrndt, P. Jonas, Boundary value problems with local generalized Nevanlinna functions in the boundary condition, Integral Equations Operator Theory 55 (2006), 453-475. MR2250158 (2008d:47079)

[5] Yu.M. Berezanskii, Expansions in Eigenfunctions of Selfadjoint Operators, Naukova Dumka, Kiev; English translation: Translations of Mathematical Monographs 17, American Mathematical Society, Providence, RI, 1968. MR0222718 (36:5768)

[6] V.A. Derkach, On Weyl function and generalized resolvents of a Hermitian operator in a Kreı̆n space, Integral Equations Operator Theory 23 (1995), 387-415. MR1361051 (96k:47062)

[7] V.A. Derkach, On generalized resolvents of Hermitian relations in Krein spaces, J. Math Sciences (New York) 97 (1999), 4420-4460. MR.1728871 (2001c:47042)

[8] V.A. Derkach, M.M. Malamud, Generalized resolvents and the boundary value problems for Hermitian operators with gaps, J. Funct. Anal. 95 (1991), 1-95. MR1087947 (93d:47046)

[9] V.A. Derkach, M.M. Malamud, The extension theory of Hermitian operators and the moment problem, J. Math. Sci. (New York) 73 (1995), 141-242. MR1318517 (95m:47009)

[10] V.A. Derkach, S. Hassi, M.M. Malamud, H.S.V. de Snoo, Generalized resolvents of symmetric operators and admissibility, Methods Funct. Anal. Topology 6, no. 3 (2000), 24-55. MR1903120 (2003b:47042)

[11] V.A. Derkach, S. Hassi, M.M. Malamud, H.S.V. de Snoo, Boundary relations and orthogonal coupling of symmetric operators, Proceedings of the Algorithmic Information Theory Conference, University of Vaasa, Finland, no. 124 (2005), 41-56. MR2222445 (2007b:47024)

[12] V.A. Derkach, S. Hassi, M.M. Malamud, H.S.V. de Snoo, Boundary relations and their Weyl families, Trans. Amer. Math. Soc. 358, no. 12 (2006), 5351-5400. MR2238919 (2007d:47026)

[13] V.A. Derkach, S. Hassi, M.M. Malamud, H.S.V. de Snoo, Boundary relations and generalized resolvents of symmetric operators, preprint, arXiv:math/0610299. To appear in Russ. J. Math. Phys.

[14] A. Dijksma, H. Langer, H.S.V. de Snoo, Eigenvalues and pole functions of Hamiltonian systems with eigenvalue depending boundary conditions, Math. Nachr. 161 (1993), 107-154. MR1251013 (94m:47091)

[15] A. Dijksma, H.S.V. de Snoo, Symmetric and selfadjoint relations in Krě̆n spaces. II, Ann. Acad. Sci. Fenn. Ser. A I Math. 12 (1987), 199-216. MR951970 (89h:47052)

[16] V.I. Gorbachuk, M.L. Gorbachuk, Boundary Value Problems for Operator Differential Equations, Kluwer Academic Publishers, Dordrecht, 1991. MR1190695 (93e:00017)

[17] S. Hassi, M. Kaltenbäck, H.S.V. de Snoo, The sum of matrix Nevanlinna functions and selfadjoint extensions in exit spaces, Oper. Theory Adv. Appl. 103, Birkhäuser, Basel, 1998, 137-154. MR1635009 (99i:47043)

[18] S. Hassi, H.S.V. de Snoo, H. Woracek, Some interpolation problems of Nevanlinna-Pick type. The Krĕn-Langer method, Oper. Theory Adv. Appl. 106, Birkhäuser, Basel, 1998, 201-216.

[19] M. Kaltenbäck, H. Woracek, On representations of matrix valued Nevanlinna functions by u-resolvents, Math. Nachr. 205 (1999), 115-130. MR.1709165 (2000f:47027)

[20] M.G. Krĕn, G.K. Langer: The defect subspaces and generalized resolvents of a Hermitian operator in the space $\Pi_{\kappa}$, Funktcional. Anal. i Prilozhen. 5 (1971), no. 2, 59-71; 5 (1971), no. 3, 54-69 (Russian); English transl.: Funct. Anal. Appl. 5 (1971/1972), 139-146 and 217228. MR0282238(43:7951a) MR 0282239(43:7951b)

[21] M.G. Kreйn, H. Langer, Uber die Q-Funktion eines $\pi$-hermiteschen Operators im Raume $\Pi_{\kappa}$, Acta. Sci. Math. (Szeged) 34 (1973), 191-230; Siberian Math. J. 18 (1977), 728-746. MR0318958 (47:7504) 
[22] M.G. KreĬn, H. Langer, Über einige Fortsetzungsprobleme, die eng mit der Theorie hermitescher Operatoren im Raume $\Pi_{\kappa}$ zusammenhängen. I. Einige Funktionenklassen und ihre Darstellungen, Math. Nachr. 77 (1977), 187-236. MR0461188 (57:1173)

[23] M.G. Krĕn, H. Langer, Some propositions on analytic matrix functions related to the theory of operators in the space $\Pi_{\kappa}$, Acta Sci. Math. (Szeged) 43 (1981), 181-205. MR621369 (82i:47053)

[24] H. Langer, B. Textorius, On generalized resolvents and Q-functions of symmetric linear relations (subspaces) in Hilbert space, Pacific J. Math. 72 (1977), 135-165. MR0463964(57:3902)

[25] H. Langer, Spectral functions of definitizable operators in Kreĭn spaces, Functional Analysis, Proceedings of a Conference held at Dubrovnik, Yugoslavia, November 2-14, 1981, Lecture Notes in Mathematics 948 (pp. 1-46), Springer-Verlag, Berlin-Heidelberg-New York, 1982. MR672791 (84g:47034)

[26] H. Langer, B. Najman, C. Tretter, Spectral theory of the Klein-Gordon equation in Pontryagin spaces, Comm. Math. Phys. 267 (2006), 159-180. MR2238908 (2007g:47032)

[27] M.M. Malamud, S.M. Malamud, Spectral theory of operator measures in a Hilbert space, Algebra i Analiz 15 (2003), 1-77; translation in St. Petersburg Math. J. 15, no. 3 (2004), 323-373. MR2052164(2005i:47008)

Department of Mathematics MA 6-4, Technische Universität Berlin, Strasse des 17. Juni 136, 10623 Berlin, Germany

E-mail address: behrndt@math.tu-berlin.de 